Revue des patrimoines

$27 \mid 2015$

Le cheval et ses patrimoines (2e partie)

\title{
Un fonds documentaire en maréchalerie
}

\section{Patrick Doffémont}

\section{(2) OpenEdition}

Journals

Édition électronique

URL : http://journals.openedition.org/insitu/12274

DOI : 10.4000/insitu. 12274

ISSN : 1630-7305

Éditeur

Ministère de la Culture

Référence électronique

Patrick Doffémont, "Un fonds documentaire en maréchalerie », In Situ [En ligne], 27 | 2015, mis en ligne le 02 novembre 2015, consulté le 14 novembre 2019. URL : http://journals.openedition.org/ insitu/12274; DOI : 10.4000/insitu.12274

Ce document a été généré automatiquement le 14 novembre 2019.

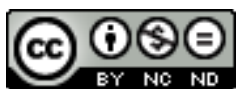

In Situ Revues des patrimoines est mis à disposition selon les termes de la licence Creative Commons Attribution - Pas d'Utilisation Commerciale - Pas de Modification 4.0 International. 


\title{
Un fonds documentaire en maréchalerie
}

\author{
Patrick Doffémont
}

\section{L'origine $^{1}$}

1 Tout a commencé par hasard en 1980, au Salon du cheval de Paris où, pris d'un coup de folie, j'ai acheté mon premier livre sur la maréchalerie. Coup de folie non pas de l'avoir acheté, mais d'y avoir laissé un mois de salaire. Ce que je prenais pour un livre très rare n'était en fait qu'un "Garsault » de 1770, la 4 e édition d'une série de dix ${ }^{2}$ (fig. 1). Je n'y ai pas appris grand-chose tant les termes, méthodes et mesures étaient pour moi incompréhensibles, mais cela m'a donné envie de constituer une bibliothèque professionnelle, d'autant plus qu'au début des années 1980, il n'y avait que deux ou trois livres techniques sur le marché. Si la recherche d'avant l'ère d'Internet était fastidieuse (trouver les librairies spécialisées, écrire, se déplacer...), celle d'après l'ère d'Internet s'est vite révélée onéreuse tant les occasions d'acheter ont considérablement augmenté, et les prix avec. 
Figure 1

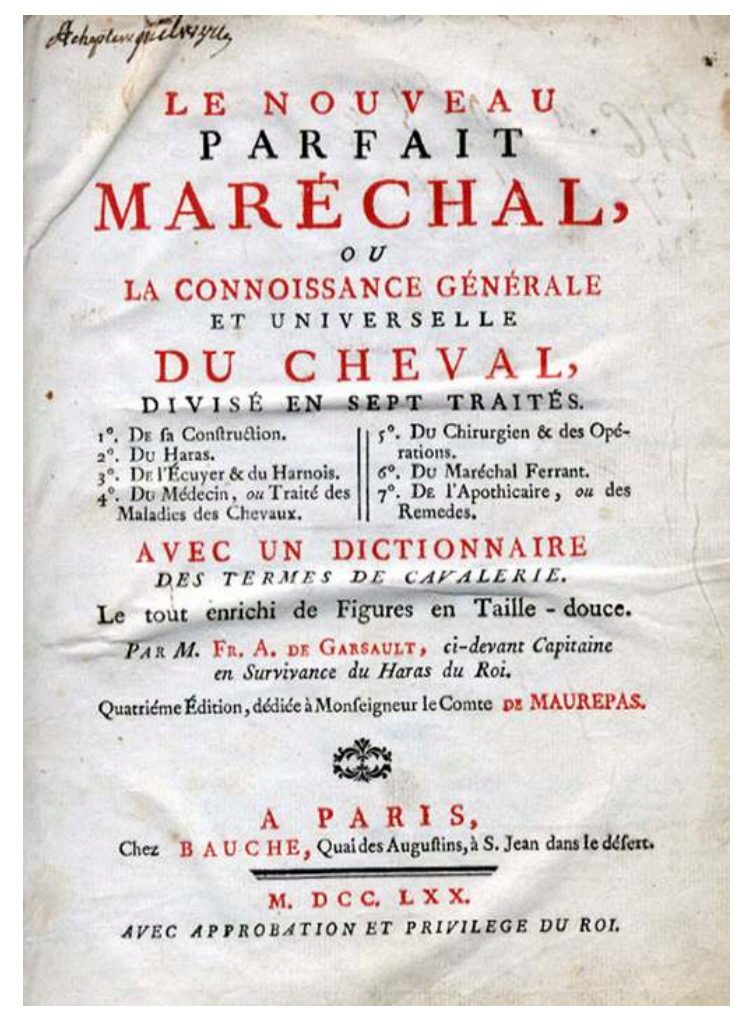

Première page du livre de François Garsault, 1770.

(c) Institut de la maréchalerie.

\section{Le contenu}

2 Trente ans après, cette bibliothèque comprend mille références, ce qui peut paraître peu pour une bibliothèque mais qui, rapporté à un métier artisanal comme celui de maréchal-ferrant est déjà intéressant. La maréchalerie résulte, aujourd'hui encore, de l'addition de deux grands domaines de compétences, celui lié aux connaissances du cheval, celles mises à jour par la science vétérinaire mais aussi celles permettant de comprendre les différents usages du cheval, privés et militaires. L'autre domaine concerne le travail du métal, en particulier à la forge : à l'évidence, la bibliothèque se devait se suivre ce schéma. C'est pourquoi elle est composée à $40 \%$ de documents sur la maréchalerie proprement dite, du début du XVII ${ }^{\mathrm{e}}$ siècle à aujourd'hui, les $60 \%$ restant se partageant entre la connaissance du cheval, le travail du fer et le contexte social et économique dans lesquels a exercé le maréchal au fil des époques, car comprendre les caractéristiques techniques de la ferrure du cheval de mine, par exemple, est impossible sans connaître ce qu'il se passe au fond.

\section{Les supports}

Cette recherche s'est très vite étendue à d'autres supports que le livre, tous véritables témoins du quotidien des collègues d'hier. Ce fonds documentaire comporte donc des journaux professionnels (une masse de données considérables puisque dans le premier tiers du $\mathrm{xx}^{\mathrm{e}}$ siècle, plusieurs titres paraissent trois fois par mois), des cartes de visite, 
des catalogues fournisseurs, également très riches d'informations, des affiches, des publicités (fig. 2), des gravures et, essentiellement sur support numérique, des enregistrements audio et toute une iconographie.

Figure 2

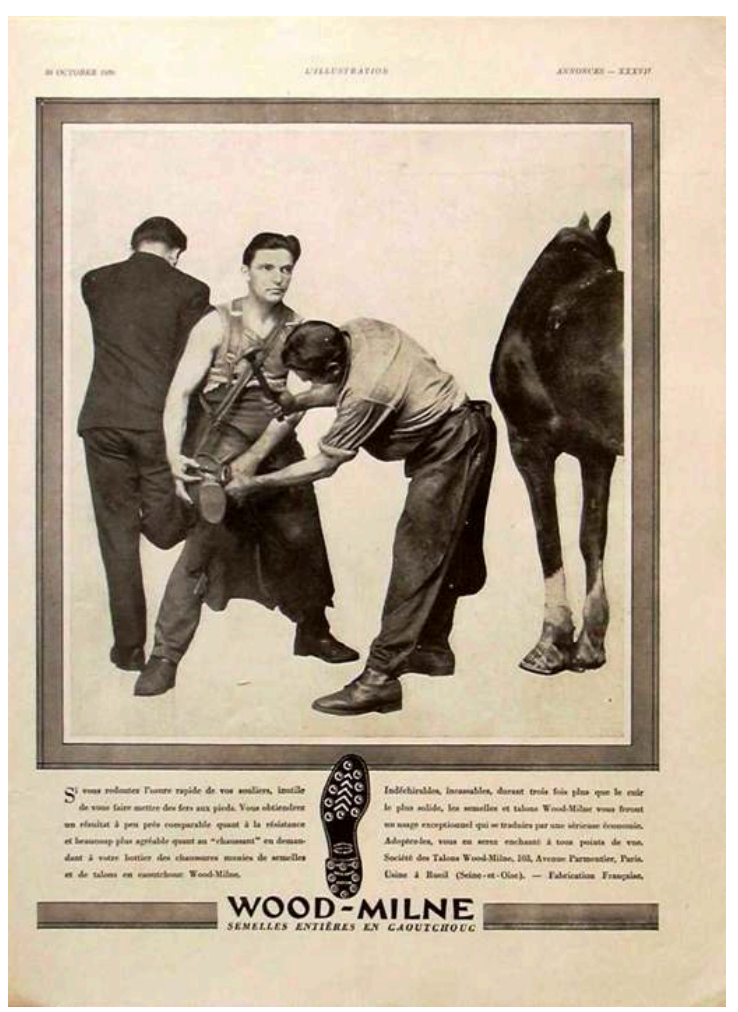

Publicité des années 1930 pour des semelles de chaussures utilisant l'image du maréchal-ferrant.

(c) Institut de la maréchalerie.

On trouve bien sûr quantité de cartes postales anciennes, mais dont j'ai dû apprendre à me méfier et à ne pas prendre pour argent comptant tout ce qu'elles représentent. En effet, on trouve parfois deux clichés identiques mais avec une légende différente, ou encore une scène posée où l'on néglige le détail technique (par exemple où le maréchal s'apprête à poser un antérieur droit sur un postérieur gauche...).

5 Le fonds comporte aussi des films, dont le plus ancien date de 1896. Mais le plus intéressant est celui de 1923, tourné dans l'école de maréchalerie de Paris. C'est un film de propagande, selon les termes de l'époque, réalisé par le syndicat des maréchaux pour inciter les jeunes à faire ce métier. De façon presque pathétique, le métier de maréchal-ferrant, qui se sait condamné mais refuse de l'admettre, utilise un moyen de communication ultra-moderne pour attirer les jeunes. Dans leurs moindres détails, ces images sont d'une extrême richesse.

Et enfin une collection de reproductions d'une centaine de tableaux, évidemment en format numérique. L'analyse de chacun de ces tableaux nous donne de nombreuses informations et parfois nous interpelle, comme celui de Jules Voirin (1833-1898) où l'on voit une scène rarement évoquée : le maréchal-ferrant reconduisant le cheval ferré de neuf chez son propriétaire, un service commercial souvent proposé. Certes, le peintre a fait du maréchal son sujet central, mais pourquoi l'a-t-il mis dans l'ombre alors que tout le reste de la scène est en pleine lumière? 


\section{La chronologie du métier}

Bref, ce fonds documentaire est constitué de tout ce qui peut donner le moindre indice permettant de retracer l'histoire de ces artisans et de ces militaires, autrement dit de tout ce qui a fait leur quotidien. Toute cette masse ( $600 \mathrm{~kg}$ de documents physiques) m'a permis de mieux comprendre la situation actuelle de la profession. Mais avant de vous dire ce que j'y ai découvert, sans doute convient-il de faire un bref résumé chronologique de l'évolution du métier (fig. 3).

1463 : enregistrement de la première corporation connue de maréchaux à Paris

1564 : premier livre où un chapitre entier est consacré à la ferrure du cheval (Fiaschi $\left.{ }^{3}\right)$

1762 : création de la première école vétérinaire à Lyon

1826 : création de l'école de maréchalerie de Saumur (114 ans d'existence !)

21950 : fin de la maréchalerie (sauf pour les courses)

31970 : début de ce que l'on appelle la démocratisation de l'équitation

41980 : première édition du championnat du monde de maréchalerie

151983 : renouveau des concours de maréchalerie en France

61985 : réapparition de la presse professionnelle

171997 : naissance de la fédération européenne des associations de maréchalerie

Figure 3

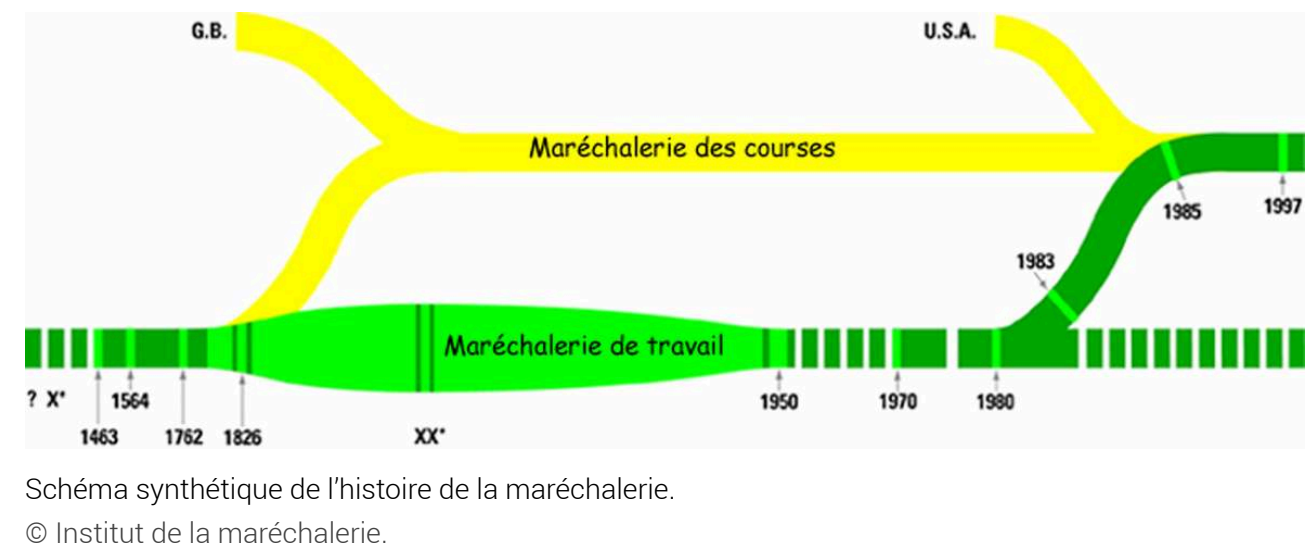

Dans ce parcours atypique, deux éléments sont essentiels. On a d'un côté une longue histoire linéaire, porteuse d'un patrimoine technique et culturel gigantesque, et de l'autre côté une absence de transmission pendant une bonne trentaine d'années. Retrouver ce patrimoine professionnel et combler autant que possible cette absence de transmission m'a aidé à comprendre la situation actuelle de notre métier.

\section{D'hier à aujourd'hui}

$19 \mathrm{Au}$ travers de cette quête, j'ai pu mettre en évidence deux points essentiels et historiques pour comprendre la situation de la maréchalerie d'aujourd'hui : 
20 - depuis dix siècles, le fonctionnement de l'appareil locomoteur est organisé pour travailler en traction, pendant des heures, sur des sols peu pénétrables et plutôt sur des trajectoires droites. Aujourd'hui il doit répondre à un travail monté, pendant des instants brefs mais intenses, sur des sols pénétrables et tournant sans cesse : l'appareil locomoteur ne fonctionne pas du tout de la même manière, et en plus d'adapter la section des fers, il nous oblige à reconsidérer tout son fonctionnement biomécanique ;

21 - depuis dix siècles, c'est le cheval qui vient à l'atelier, la forge est un lieu social fort (Fernand Braudel l'a qualifié de "lavoir des hommes", mais aujourd'hui c'est le maréchal qui se déplace avec son véhicule atelier vers ses clients. Le maréchal est sorti de son atelier, ce qui ne manque pas de modifier les relations professionnelles.

22 Le maréchal-ferrant réapparaît donc, mais dans un autre contexte, un autre décor. Tout a changé. Le rapport à l'animal, car le cheval, hier de compagnon de travail, avec parfois ses abus, est aujourd'hui compagnon de loisir avec aussi ses abus, entre autres ses carences en matière d'éducation, ce qui rajoute à la pénibilité du métier. L'histoire continue et la maréchalerie s'adapte aux besoins de la filière équine (fig. 4).

Figure 4

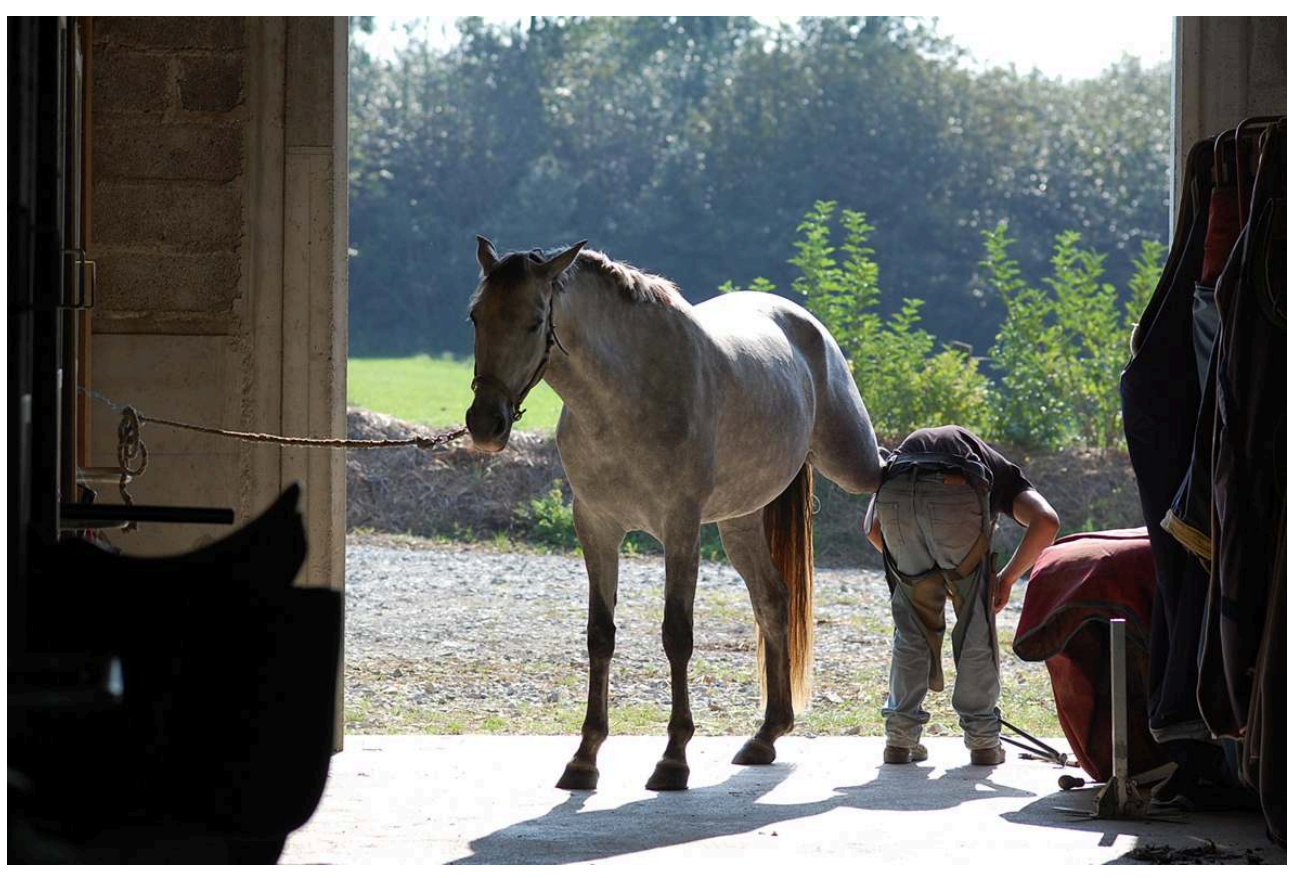

La maréchalerie actuelle au service du cheval de sport et de loisir.

(c) Institut de la maréchalerie.

\section{La transmission}

23 Le problème de la succession ou du devenir de cette collection est déjà réglé pace que j'ai tout cédé à l'Association ouvrière des Compagnons du Devoir et du Tour de France ${ }^{4}$. Je ne me suis jamais senti propriétaire de ce fonds car même si j'ai consacré beaucoup d'efforts pour le constituer, je n'ai fait que rassembler ce qui appartient au métier, c'est-à-dire aux hommes et aux femmes de métier d'hier, d'aujourd'hui et de demain. C'est pourquoi il appartient à ceux qui nous suivent de continuer à alimenter ce fonds d'autres trouvailles anciennes à dénicher, certes, mais aussi de tout ce qui fait 
l'actualité du métier. L'ensemble de ce fonds est consultable sur rendez-vous à Angers, au Centre de la mémoire des Compagnons du Devoir, association de compagnonnage à laquelle j'appartiens et pour laquelle je travaille à préparer le mieux possible les professionnels de demain.

\section{NOTES}

1. - Cette intervention a été faite par Jean Mopin le 4 octobre 2012. Ce dernier ayant quitté ses fonctions, ce texte a été relu et validé par Patrick Doffémont de l'institut de la maréchalerie. Centre de la mémoire des Compagnon du Devoir (sur rendez-vous). 3 bd Marc-Leclerc, 49100 Angers (02 418864 97, archives@compagnons-du-devoir.com).

2. - GARSAULT, François-Alexandre de. Le nouveau parfait maréchal, ou La connaissance générale et universelle du cheval. Paris : $1^{\mathrm{e}}$ éd. 1741 , chez Charles Leclerc.

3. - FIASCHI, Cesare. Trattato dell'imbrigliare, atteggiare e ferrare cavalli. $1^{\mathrm{e}}$ éd. 1556.

4. - Voir le site : http://www.compagnons-du-devoir.com.

\section{RÉSUMÉS}

Constituer un fonds documentaire dédié au métier de maréchal-ferrant permet de rassembler tout ce qui a fait son histoire et de transmettre l'important patrimoine technique et culturel qu'il s'est constitué au fil des siècles. Il aide à combler l'absence de transmission des années 1950-1970 et à comprendre la situation actuelle. Mais il sert surtout à préparer l'avenir car anticiper, et donc organiser la formation des jeunes en conséquence, impose d'être au moins informé, sinon imprégné, de tout ce qui fait son identité et son rôle social.

Documenting the blacksmith's trade. The constitution of a documentary collection devoted to the blacksmith's and farrier's trade allows for the history of this trade, which comprises an important technical and cultural heritage built up over the centuries, to be transmitted to younger generations. The documentation also helps understand the absence of this transmission between the 1950s and 1970s and to appreciate the situation today. Above all, however, it is necessary for the future, for training of young people in the farrier's trade, and for understanding his identity and role in society.

\section{INDEX}

Mots-clés : maréchal-ferrant, maréchalerie, ferrure, fer à cheval

Keywords : farrier, blacksmith, horse shoe 
AUTEUR

\section{PATRICK DOFFÉMONT}

Institut de la maréchalerie im@compagnons-du-devoir.com 